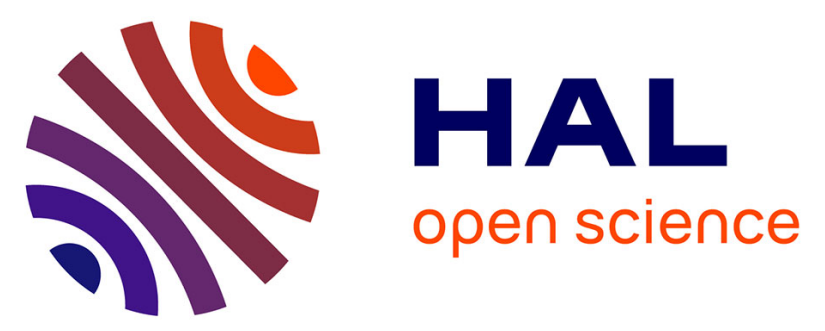

\title{
Crucial Role of Conjugation in Monolayer-Protected Metal Clusters with Aromatic Ligands: Insights from the Archetypal Au_144L_60 Cluster Compounds
}

Rajarshi Sinha-Roy, Xóchitl López-Lozano, Robert Whetten, H.C. Weissker

\section{- To cite this version:}

Rajarshi Sinha-Roy, Xóchitl López-Lozano, Robert Whetten, H.C. Weissker. Crucial Role of Conjugation in Monolayer-Protected Metal Clusters with Aromatic Ligands: Insights from the Archetypal $\mathrm{Au}$ _144L_60 Cluster Compounds. Journal of Physical Chemistry Letters, 2021, 12 (38), pp.92629268. 10.1021/acs.jpclett.1c02597 . hal-03427440

\author{
HAL Id: hal-03427440 \\ https://hal.science/hal-03427440
}

Submitted on 13 Nov 2021

HAL is a multi-disciplinary open access archive for the deposit and dissemination of scientific research documents, whether they are published or not. The documents may come from teaching and research institutions in France or abroad, or from public or private research centers.
L'archive ouverte pluridisciplinaire HAL, est destinée au dépôt et à la diffusion de documents scientifiques de niveau recherche, publiés ou non, émanant des établissements d'enseignement et de recherche français ou étrangers, des laboratoires publics ou privés. 


\section{Crucial Role of Conjugation in}

\section{Monolayer-protected Metal Clusters With}

\section{Aromatic Ligands: Insights from the Archetypal $A u_{144} \mathbf{L}_{60}$ Cluster Compounds}

Rajarshi Sinha-Roy, ${ }^{*, \dagger, t, \uparrow, \perp}$ Xóchitl López Lozano, ${ }^{\S}$ R.L. Whetten, $\|$ and Hans-Christian Weissker ${ }^{*, \dagger, \perp}$

$\dagger$ †ix-Marseille Univ., CNRS, CINAM, Marseille, France.

$\ddagger$ Departamento de Física Teórica de la Materia Condensada and Condensed Matter Physics Center (IFIMAC), Universidad Autónoma de Madrid, E-28049 Madrid, Spain

ILaboratoire des Solides Irradiés, Ecole Polytechnique, CNRS, CEA, Université Paris-Saclay, Palaiseau 91128, France

$\S$ Department of Physics 83 Astronomy, The University of Texas at San Antonio, One UTSA circle, 78249-0697 San Antonio, TX., USA

|| Department of Applied Physics and Materials Science, and MIRA, Northern Arizona University, Flagstaff, Arizona 86011, USA $\perp$ European Theoretical Spectroscopy Facility (ETSF)

E-mail: rajarshi.sinha-roy@polytechnique.edu; weissker@cinam.univ-mrs.fr 


\begin{abstract}
Ligand-protected metal clusters are employed in a great many applications that include notably energy conversion in a prospective hydrogen-based energy supply. The interaction between the ligands and the metallic cores, mediated by an often complex interface, influences profoundly the properties of in particular small clusters. Nonetheless, the mechanisms of interaction remain far from fully understood.

The $\mathrm{Au}_{144} \mathrm{~L}_{60}$ class of cluster compounds has long played a central role in the study of monolayer-protected clusters, but total structure determination has been achieved only recently for a thiolated and an all-alkynyl cluster. Both ligands contain aromatic rings but differ in their ligation to the metal core: conjugation along a triple bond in the former, saturation in the latter.

Studying the two closely analogous crystallographically determined structures as well as an ad hoc saturated structure, we demonstrate the paramount importance of the conjugation for the electronic and optical properties of monolayer-protected metal clusters.
\end{abstract}

\title{
Introduction
}

Noble-metal clusters have attracted interest in view of energy conversion for a hydrogenbased energy supply: thiolate-protected gold clusters are studied as electrocatalysts for energy conversion, as cocatalysts of water-splitting photocatalysts, and as photosensitizers in solar cells. ${ }^{1}$ Other uses comprise protein tagging, ${ }^{2}$ biomolecule labeling, ${ }^{3}$ inhibition of HIV fusion, ${ }^{4}$ and antibacterial and antifungal action. ${ }^{5,6}$

In recent years, total structure determination has been accomplished of many atomically precise clusters ${ }^{7,8}$ of which complex ligand-metal interfaces make up a substantial part. Their optical properties are particularly interesting because these are exploited in many of the applications, but also because they reflect the complex dynamic coupling between the metal core and the ligands. However, the understanding of the role of ligandcore interaction remains far from complete. 
The class of (icosahedral) cluster compounds denoted $\mathrm{Au}_{144} \mathrm{~L}_{60}$ are special among the monolayer-protected clusters owing to the high stability of, in particular, the thiolated cluster compound that was produced and studied by many groups. ${ }^{9}$ However, it wasn't until 2018 that total structure determination has been accomplished: one version with thiolate ligands, $\mathrm{Au}_{144}\left(\mathrm{SCH}_{2} \mathrm{Ph}\right)_{60}{ }^{10}$ and one all-alkynyl compound $\mathrm{Au}_{144}(\mathrm{C} \equiv \mathrm{CAr})_{60}$ with $\mathrm{Ar}=2-\mathrm{FC}_{6} \mathrm{H}_{4^{-}} .{ }^{11}$ The coordination of the Au atoms is identical between these structures, and likewise identical to the $\operatorname{model}(\mathrm{s})$ worked out earlier. ${ }^{12,13}$ In the following, we refer to these two structures as "the (full) thiolate" and "the (full) alkynyl" clusters.

A multitude of individual peaks pervades the VIS and near-UV optical spectra of $\mathrm{Au}_{144} \mathrm{~L}_{60}{ }^{14}$ which are much more peaked than those of clusters of comparable size but lower symmetry. ${ }^{7}$ Together with the fact that $\mathrm{Au}_{144}$ compounds can be synthesized with many different ligands, this makes them an ideal benchmark to study the influence of different ligands on the optical properties. Earlier comparisons of different measurements seemed to indicate that the impact of different ligands is rather limited. ${ }^{15}$ However, the absorption spectra of the two structure-determined clusters are strikingly distinct. ${ }^{10,11,16}$

It was shown early on ${ }^{12}$ that the $\mathrm{Au}_{144}$ clusters show the characteristic frontier-orbital structure of "Super-Atom Complexes" (SAC). ${ }^{17}$ However, the calculation of optical spectra was accomplished only much later, hindered by the sheer immensity of the systems and necessitating different ligand simplifications in the thiolates $\mathrm{SR}$ like $\mathrm{R}=\mathrm{H},{ }^{18,19}$ or $\mathrm{R}=\mathrm{CH}_{3} \cdot{ }^{14}$

The question whether such ligand simplification is adequate ${ }^{15,20}$ rejoins the physicochemical question about the influence of the ligands on the clusters' properties. However, until recently neither the computational means nor experimentally confirmed structures including the full ligands were available to conclusively answer these interrogations. Instead, investigations have focused on smaller systems of lower symmetry (greater interfacial complexity). Fortunelli and coworkers have pointed out that aromatic ligands have a greater influence on the optical properties of small gold clusters $\left(\mathrm{Au}_{36}, \mathrm{Au}_{38}, \ldots\right)$ than 
aliphatic ligands, ${ }^{21-24}$ the effects were discussed in terms of electron delocalization, ${ }^{25}$ and it was noted that the influence may extend through energies below the absorption features of the isolated ligands. The aromatic rings were directly connected to the "staples," as in $\mathrm{Au}_{38}(\mathrm{SPh})_{24} \cdot{ }^{23}$ However, it is not clear how these pieces of information carry over to the critical size of incipient plasmonic / metallic cores. ${ }^{7,14,18}$

In the structure-determined thiolate and alkynyl $\mathrm{Au}_{144}$ clusters, both ligands, shown in Fig. 1 (a,b) have an aromatic carbon ring which is connected to two gold atoms via a bridge of two atoms. Two main differences exist: (i) the unsaturated $-\mathrm{C} \equiv \mathrm{C}-$ triple bond along this connection in the alkynyl vs. the saturated sulfur-carbon single bond $\left(-\mathrm{S}-\mathrm{CH}_{2}-\right)$ in the thiolate ligand; (ii) the presence of $\mathrm{C}$ atoms in the alkynyl vs. $\mathrm{S}$ atoms in the thiolate "staples."

We will show in the present Letter that it is, in fact, the conjugation provided by the triple bond in the alkynyl ligands that causes the most profound effects.

The full clusters have been relaxed starting from the experimental coordinates. In the alkynyl ligands, the fluorine atom was replaced by hydrogen as in the experimental Ref. 16. Shown in Fig. 1 (a,b), both structures including the full ligands exhibit I symmetry (60 point group operations, equivalence of all 60 ligands). Details are discussed in the Supplementary Information.

Electronic structure. The $\mathrm{Au}_{144}$ clusters show clear SAC behavior. ${ }^{12}$ Compared with the thiolate structure in Fig. 1 (c), a striking difference is the much larger HOMOLUMO gap of $0.56 \mathrm{eV}$ of the alkynyl structure, between a five-fold degenerate $\mathrm{D}$ state $(L=2)$ and five $\mathrm{H}$ states $(L=5)$. It is striking that the thiolate cluster's SAC states look similar: the same D and H states that make up HOMO and LUMO of the alkynyl structure are present at almost exactly the same energetic distance (arrows). However, on finds two very close-lying states, one of $\mathrm{S}(L=0)$ and one of I $(=6)$ character, mid- 

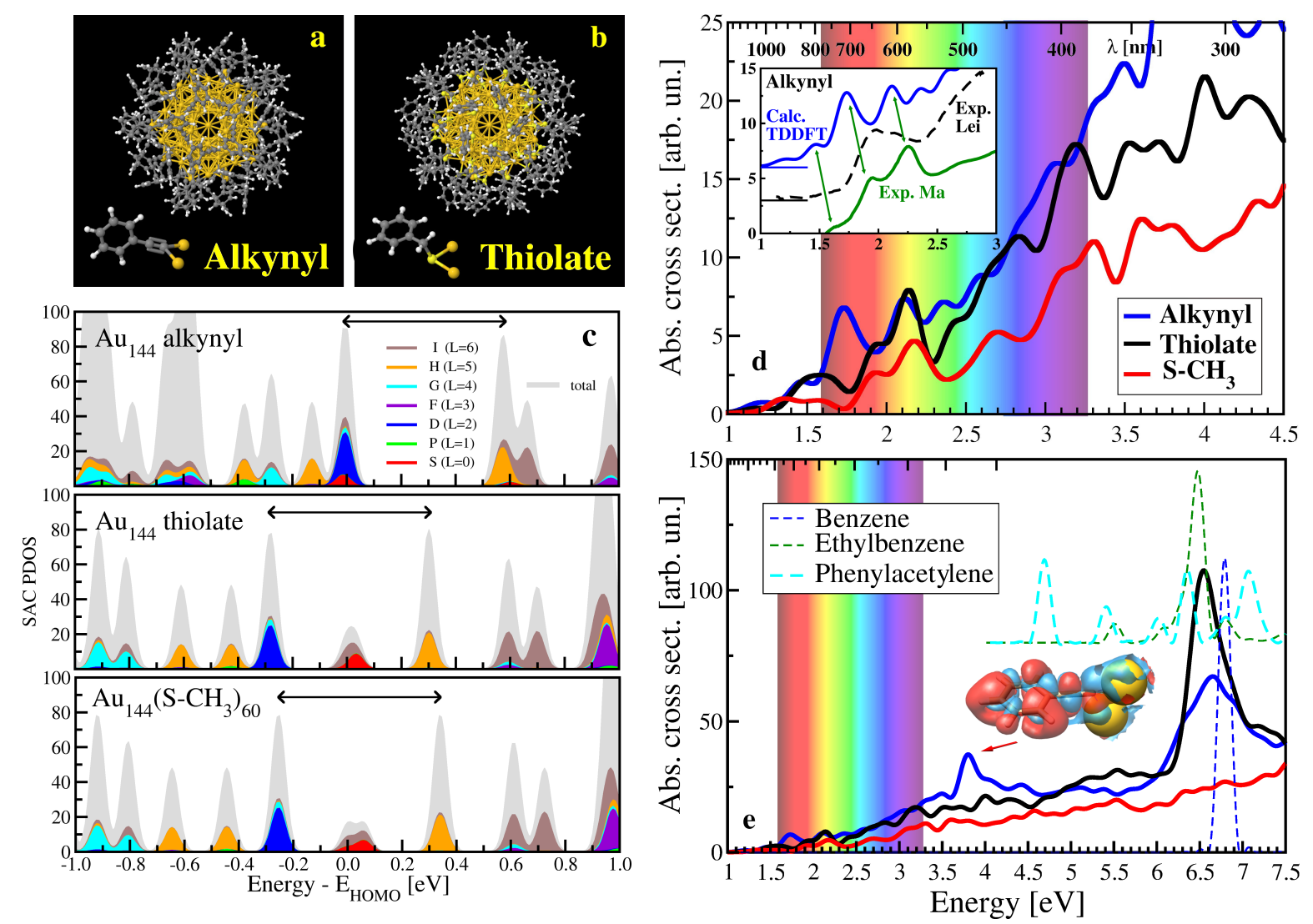

Figure 1: (a,b) Cluster structures after relaxing the crystallographically determined full clusters, highlighting the differences: the $-\mathrm{C} \equiv \mathrm{C}-$ triple bond vs. $-\mathrm{S}-\mathrm{CH}_{2}-$ single bond, and C vs. S in the "staples." (c) SAC projected density of states. (d) TDDFT spectra in the VIS range, comparing the full clusters and the truncated previously used model from Refs. $[13,14]$ where $\mathrm{R}=\mathrm{CH}_{3}$. Inset: comparison with experiments from Refs. $[11,16]$. (e) larger energy range and comparison with the isolated ligand molecules. Inset: induced density around one alkynyl ligand corresponding to the peak at $3.8 \mathrm{eV}$ (cf., Fig. 2).

way between the $\mathrm{D}$ and the $\mathrm{H}$ states. This produces the smaller gap. We note that the PDOS of the simplified $\mathrm{Au}_{144}\left(\mathrm{SCH}_{3}\right)_{60}$ matches well that of the full thiolate cluster in the vicinity of the gap. This has a bearing on our discussion of the spectra below.

The spectra of the alkynyl and the thiolate clusters shown in Fig. 1(d,e) are very different in the VIS energy range. In particular, a pronounced peak in the alkynyl structure's spectrum at about $1.7 \mathrm{eV}$ is absent in the thiolate cluster. A strong peak at high energies $(\approx 6.8 \mathrm{eV}$, Fig. $1(\mathrm{e}))$ is present in both structures when the full ligand is included. Comparison with the spectrum of free benzene, i.e., an isolated aromatic ring, suggests that this resonance originates from the ligands' aromatic rings (localized phenyl group excitation $\left(\right.$ pi-pi*) $\left.^{*}\right)$. We will show below that this is indeed the case. 

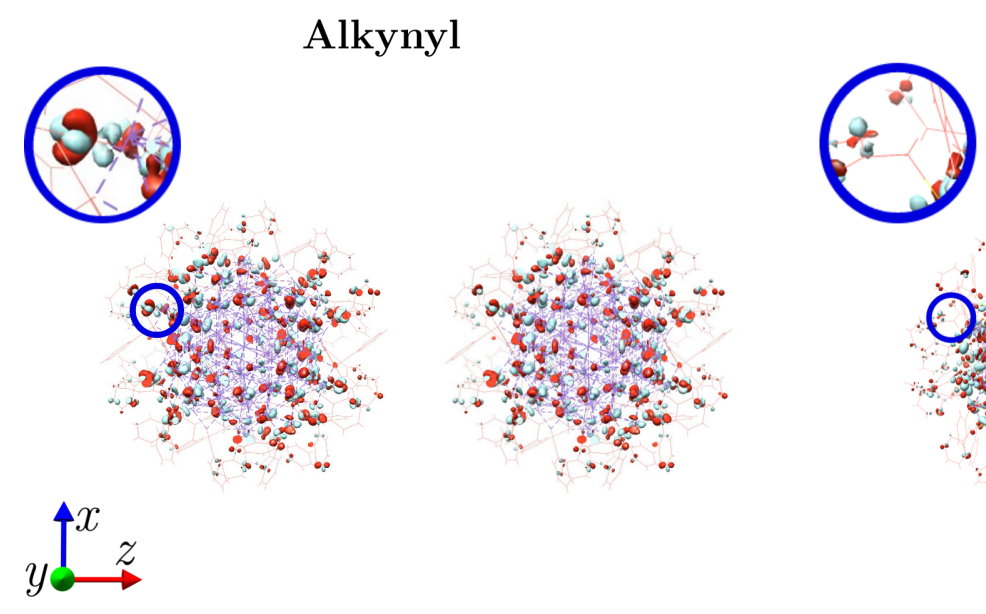

Thiolate

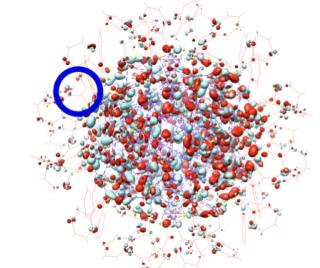

$2.13 \mathrm{eV}$; iso value $5 \mathrm{e}-7$
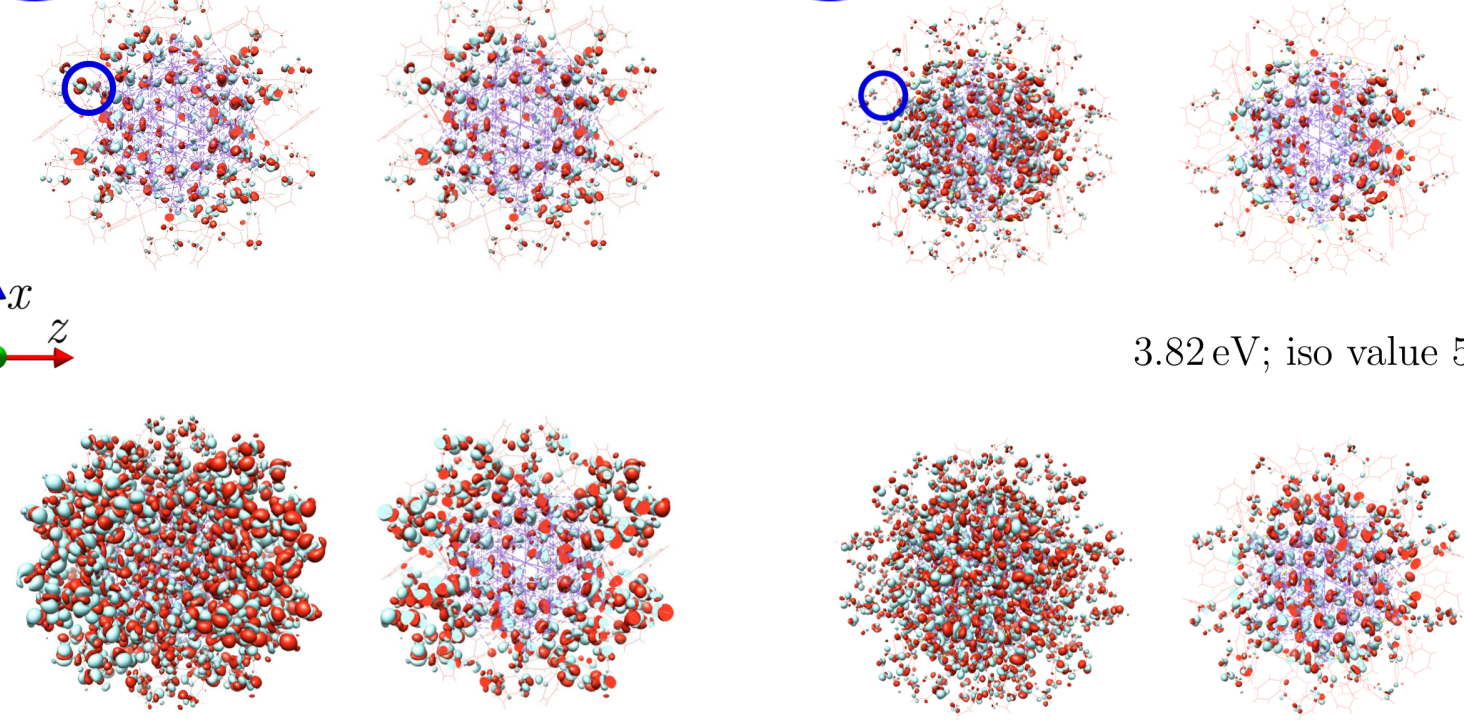

$3.82 \mathrm{eV}$; iso value $5 \mathrm{e}-7$

$6.54 \mathrm{eV}$

High-energy peak; iso value 1e-6

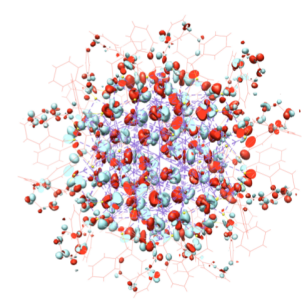

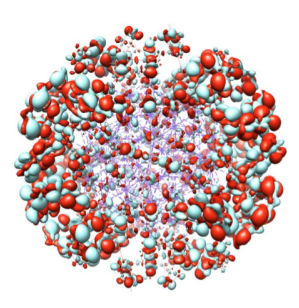

(full)

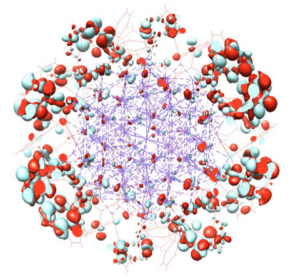

(slab @ center)

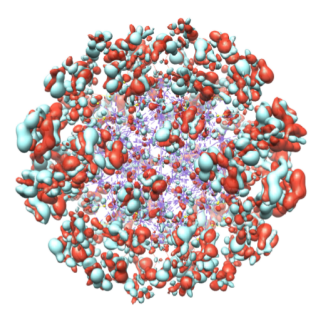

(full)

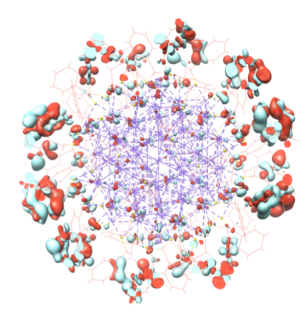

(slab@ center)
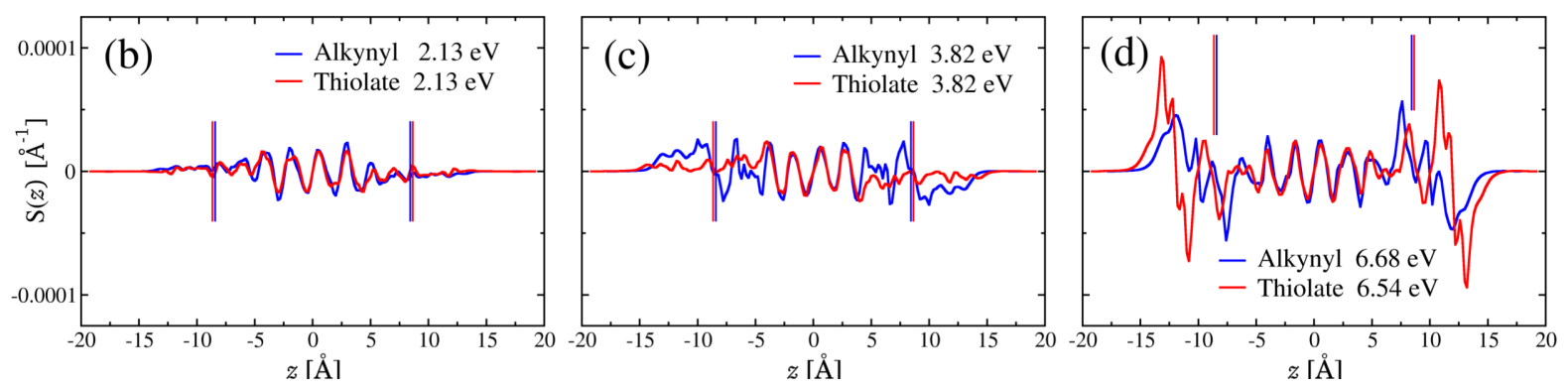

Figure 2: Induced densities for the different prominent excitations in the spectra of the complete alkynyl (left two columns) and thiolate (right two columns) clusters. For each energy and system, we show two representations: the standard isosurface representation (left figure of each group) and the same isosurface cut to a $5 \AA$ slab located symmetrically around the center of the cluster in the $y-z$ plane. Additional views are shown in Supplementary Figures 7 and 8. The blue circles mark the connections of one ligand ring to the cluster core in each of the two cases, highlighting the differences between the alkynyl structure where at the triple bond there is a contribution, and the thiolate cluster where at the saturated carbon atoms there is none. For an enlargement, see Supplementary Fig. 6. The excitation is applied along $z$, which is one of the 5-fold symmetry axes. Bottom line (b,c,d): same induced densities in integrated 1d representation (see text). Vertical lines: positions of the outermost Au atoms. 
Consequently, we arrive at the intuitive picture of the clusters' response as produced by excitations in the ligand rings, coupled to those of the core. The high-energy peak is sharper for the thiolate than for the alkynyl structure, suggesting a stronger coupling in the latter.

In addition, a strong, broad peak is seen in the near-UV at $3.82 \mathrm{eV}$ for the alkynyl structure, absent in the spectrum of the thiolate cluster. This is well below the onset $(\approx 4.6 \mathrm{eV})$ of absorption in the isolated ligand molecules (shown in Fig. 1(e)), which suggest strong coupling between the Au core and ligand rings.

Finally, the spectrum of the truncated thiolate cluster with $\mathrm{R}=\mathrm{CH}_{3}$ appears rather similar and related by a minor scaling of intensity to that of the full thiolate cluster below $2.2 \mathrm{eV}$, as expected from the similarity of the electronic structures in Fig. 1(c). The deviations become bigger for higher energies. Naturally, the high-energy peak at $6.82 \mathrm{eV}$ is absent in the truncated model. We note that our results for the thiolate cluster are naturally close to those of Ref. 26 concerning electronic structure and spectrum.

Induced densities. Fig. 2 shows the electronic modes contributing to the individual absorption features, calculated using a Fourier-transform analysis of the time-dependent density that some of the present authors recently presented and tested in detail. ${ }^{27}$ Further explanation can be found in the Supplementary Material.

The structures are included in "wireframe" representation. To further clarify our observations, Figs. 2(b-d) show one-dimensional integrated representations of the same induced densities along the direction of the excitation, $S(z)=\int d x d y[\rho(x, y, z, t)-$ $\left.\rho_{\mathrm{GS}}(x, y, z)\right]_{R<9 \mathrm{~A}^{\circ}}$.

The peak at $2.13 \mathrm{eV}$ has roughly the same intensity for both structures, so also the induced densities can be directly compared. The overall shape is a dipole extending over the clusters, while smaller local dipoles are likewise visible. In both cases, the main contribution resides inside the gold core. For the thiolate structure, this is consistent with the finding by Malola et al. that this portion of the spectrum is mainly produced by contributions from the gold and sulfur atoms. ${ }^{26}$ Small contributions from the ligand layer 
are slightly more pronounced in the alkynyl structure, suggesting a stronger coupling between the ligand layer and the core.

The most important difference is a substantial contribution located around the triple bonds in the alkynyl structure, largely absent on the saturated $-\mathrm{S}-\mathrm{CH}_{2}-$ bridge in the thiolate structure (Fig. 2 (enlargements) and Fig. S5).

The peak at $3.82 \mathrm{eV}$ of the alkynyl structure is compared with the same energy in the thiolate cluster's spectrum with no peak. There are relatively strong contributions from the alkynyl ligands, with smaller contributions inside the core. In the thiolate cluster, the contributions are almost entirely located in the core, with only very small dipoles in the ligands. Once more, this indicates a stronger coupling of the ligands' response to that of the core for the alkynyl cluster. The one-dimensional representation in Fig. 2(c) confirms this clearly.

The high-energy peak at $6.68 \mathrm{eV}$ (alkynyl) and $6.54 \mathrm{eV}$ (thiolate) derives mainly from contributions located in the aromatic rings of the ligands, thus confirmed by Fig. 2 to be modified resonances of the rings. The stronger coupling between ligand rings and metal core in the alkynyl structure, leading to a larger width of the resonance, can be seen in Fig. 2(d) which confirms the similarity of the modes in the two structures, but with a sharper induced-density profile in the thiolate cluster.

To explain the differences in the optical response of the clusters in a generalizable manner, what remains to be done is to unequivocally trace the origin of the coupling between the ligands' aromatic rings and the metal core. The principal differences between the ligands are the triple bond in the alkynyl ligand that maintains the conjugation between the aromatic rings and the core, and the presence of the sulfur atom in the thiolate ligand.

To identify the effect of the triple bond, a way is needed to switch the conjugation on and off. To this end, we have constructed an ad hoc structure by saturating the triple bonds in the alkynyl cluster with $2 \times 2=4 \mathrm{H}$ atoms, i.e., by replacing $-\mathrm{C} \equiv \mathrm{C}-\mathrm{Ph}$ by $-\mathrm{CH}_{2}-\mathrm{CH}_{2}-\mathrm{Ph}$, adjusting bond lengths and angles. The structure relaxes well and thus 


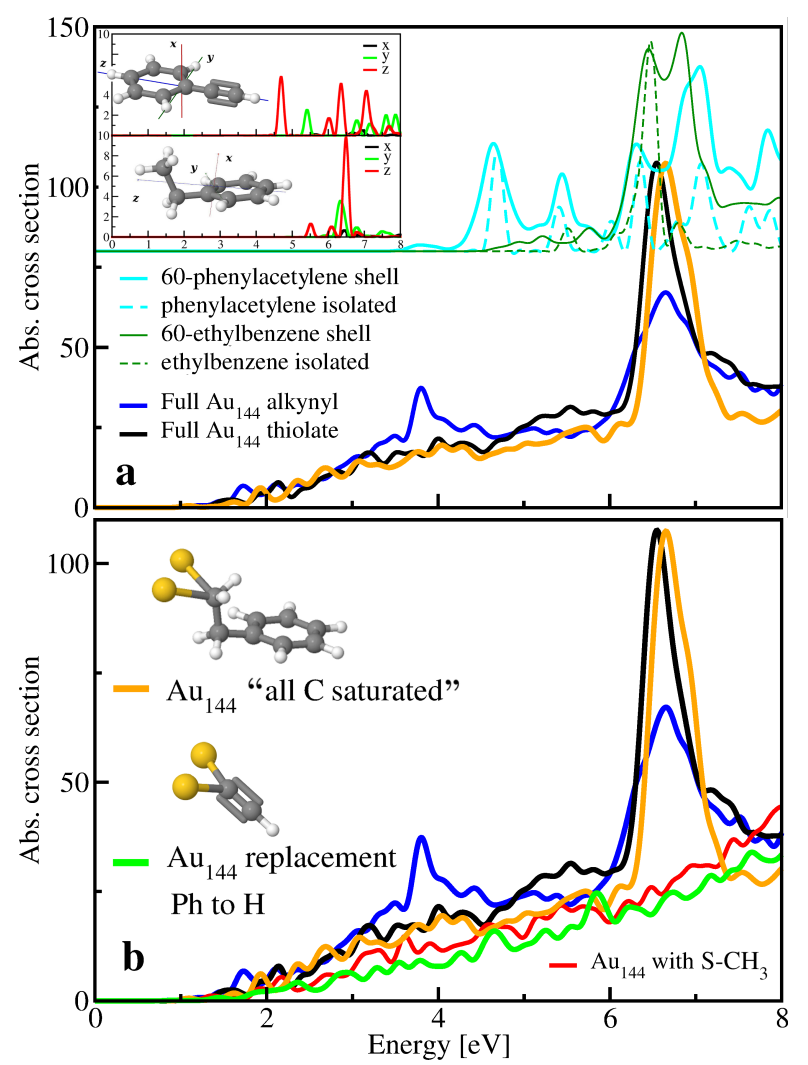

Figure 3: Switching off the conjugation: (a) Absorption spectra of the two complete clusters, thiolate and alkynyl, compared with the artificial "all-C-saturated" structure where the rings are connected by $-\mathrm{CH}_{2}-\mathrm{CH}_{2}-$ to the core, i.e., where the conjugation has been switched off. This spectrum is very similar to that of the thiolate structure without triple bond - the main effects consequently originate from the conjugation / the triple bond, not from the difference of the atoms in the staples, C vs. S. Shifted up: spectra of the isolated ligand molecules and of the ligand shells only. Inset: response of phenylacetylene and ethylbenzene molecules in the different directions, with the strong lowest peak in phenylacetylene corresponding to the long direction (z). (b) additional comparison with the effect of replacing the phenyl rings in the alkynyl structure by $\mathrm{H}$ atoms, thus retaining the triple bonds. The resulting spectrum is very close to that of the $\mathrm{S}-\mathrm{CH}_{3}$ structure; the simple presence of the triple bonds does not impact the spectra strongly.

can be studied for its optical properties as an at least metastable system.

The spectrum of the resulting "all-C-saturated" structure in Fig. 3 is indeed very close to that of the full thiolate cluster, even though there is no sulfur in it now. Even the width of the high-energy peak coincides very well with that of the thiolate cluster; the difference to the full alkynyl cluster is large. This comparison shows unequivocally that the difference is mainly due to the conjugation, whereas the presence of the sulfur atom in the $\mathrm{Au}-\mathrm{S}$ or $\mathrm{Au}-\mathrm{C}$ "staples" is far less important, although it is probably responsible for the fact that the spectrum is not entirely identical to the thiolate spectrum in the 
VIS region, in view of the above-mentioned origin of this region of the spectrum from the inorganic core (Au+S) (cf., Fig. 2 and Ref. 26).

To confirm that the differences are not simply due to the presence of the triple bond but indeed to the coupling between the ligand rings and the core, we employ another simplified model where the alkynyl ligands' phenyl rings are replaced by $\mathrm{H}$ atoms. The spectrum of the resulting $\mathrm{Au}_{144}[\mathrm{C} \equiv \mathrm{C}-\mathrm{H}]_{60}$ in Fig. 3(b) is very close to that of the truncated model with $\mathrm{R}=\mathrm{CH}_{3}$, i.e., with no carbon rings or conjugation. This shows that the mere presence of the proximal triple bonds does not influence the spectra strongly; the increased delocalization as discussed in Ref. 25 is here not sufficient to induce substantial differences.

Finally, to identify clearly the units between which the conjugation is at play, we study the overall spectra in view of the individual molecules derived from the ligands by $\mathrm{H}$ termination and replacement $\mathrm{S} \rightarrow \mathrm{C}$, so as to produce phenylacetylene and ethylbenzene. The spectra of the isolated molecules, as well as of the full shells without the gold core, are shown in Fig. 3(a).

The isolated ethylbenzene molecule produces a strong peak similar to isolated benzene, all other features are relatively weak. In other words, the aromatic ring dominates, the saturated $\mathrm{H}-\mathrm{CH}_{2}-\mathrm{CH}_{2}-$ chain contributes negligibly below $7 \mathrm{eV}$, typical for any saturated hydrocarbon, e.g., cyclohexane. This is analogous to the weak coupling of the rings to the core in the thiolate structure that leads to the close similarity of the spectra of the truncated thiolate $\left(\mathrm{R}=\mathrm{CH}_{3}\right)$ and the full thiolate clusters, despite the presence of the aromatic rings in the latter.

In the phenylacetylene molecule, there is one strong resonance at much lower energy compared to the isolated-benzene spectrum for excitation parallel to the long $(z)$ axis along which the conjugation (triple bond) provides for electron delocalization. That corresponds to the situation in elongated nanostructures ("nanorods") with delocalized electrons, where a strong resonance for excitation along the long axis is strongly redshifted with increasing aspect ratio. ${ }^{28-30}$ A similar effect is found in the series of acenes. ${ }^{31}$ 
The peak at $3.8 \mathrm{eV}$ in the alkynyl cluster spectrum thus corresponds, strongly redshifted (by $0.85 \mathrm{eV}$ ), to the lowest peak of the phenylacetylene. This is confirmed by the induced density at that energy: the insert in Fig. 1(e), extracted from the overall density shown in Fig. 2, shows the dipole shape of the density oscillation within the phenylacetylene ligand bridging the two gold atoms. Its location in the full structure is shown in Supplementary Fig. S9.

These results show clearly the effect of conjugation (i) for coupling between the ligands and the metal core, and, not surprisingly, (ii) within the aromatic ligand molecules. For the alkynyl clusters, this ligand-core coupling leads to a strong redshift and broadening of the lowest absorption peak of the isolated ligand to produce the broad peak at $3.82 \mathrm{eV}$, thus influencing the spectra at energies far below the absorption of the isolated ligands.

This type of ligand-core coupling is negligible in the full thiolate cluster, which is corroborated by the close similarity between its spectrum and that of the truncated $\mathrm{Au}_{144}\left(\mathrm{SCH}_{3}\right)_{60}$ (with the high-energy peak trivially missing).

This shows that the saturated methylene pair $\left(-\mathrm{CH}_{2}-\mathrm{CH}_{2}-\right)$ are sufficient to disrupt the coupling between the metal core and the aromatic ligand rings. Such situations, then, comprise many ligands for which the precise ligand $\mathrm{R}$ group bears little influence on the spectra. ${ }^{15}$

In conclusion, we have demonstrated the paramount importance of conjugation and of the resulting coupling along the connection between metal cores and aromatic ligand rings in monolayer-protected noble-metal clusters, with profound influence on their optical properties. The mere presence of the aromatic rings in the ligands is not sufficient to produce any such effects, their connection to the core is determinant. It should be evident that even though we have here made use of the availability of the experimental structures of $\mathrm{Au}_{144}$ with two different aromatic ligands, the comparisons and conclusions are expected to be valid for monolayer-protected metal clusters in general. 


\section{Acknowledgement}

We thank Quan-Ming Wang and Ignacio Garzón for providing and treating the coordinates of the all-alkynyl cluster compound. This work has been carried out in part thanks to the support of the A*MIDEX grant (nANR-11-IDEX-0001-02) funded by the French Government "Investissements d'Avenir" program. We acknowledge support from the French National Research Agency (Agence Nationale de Recherche, ANR) in the frame of the project "FIT SPRINGS", ANR-14-CE08-0009. This work has used HPC resources from GENCI-IDRIS (Grant 2019-096829). Moreover, HCW and RSR would like to acknowledge the contribution of the International Research Network IRN Nanoalloys (CNRS). RLW acknowledges support from the Welch Foundation (AX-1857).

\section{Conflict of interest}

There are no conflicts of interest to declare.

\section{References}

(1) Kawawaki, T.; Ebina, A.; Hosokawa, Y.; Ozaki, S.; Suzuki, D.; Hossain, S.; Negishi, Y. Thiolate-Protected Metal Nanoclusters: Recent Development in Synthesis, Understanding of Reaction, and Application in Energy and Environmental Field. Small 2021, n/a, 2005328.

(2) Hainfeld, J. F.; Liu, W.; Halsey, C. M.; Freimuth, P.; Powell, R. D. Ni-NTA-Gold Clusters Target His-Tagged Proteins. Journal of Structural Biology 1999, 127, 185 $-198$.

(3) Ackerson, C. J.; Powell, R. D.; Hainfeld, J. F. In Cryo-EM Part A Sample Preparation and Data Collection; Jensen, G. J., Ed.; Methods in Enzymology; Academic Press, 2010; Vol. 481; pp 195 - 230. 
(4) Bowman, M.-C.; Ballard, T. E.; Ackerson, C. J.; Feldheim, D. L.; Margolis, D. M.; Melander, C. Inhibition of HIV Fusion with Multivalent Gold Nanoparticles. Journal of the American Chemical Society 2008, 130, 6896-6897.

(5) Bresee, J.; Maier, K. E.; Boncella, A. E.; Melander, C.; Feldheim, D. L. Growth Inhibition of Staphylococcus aureus by Mixed Monolayer Gold Nanoparticles. Small 2011, 7, 2027-2031.

(6) Lopez, P.; Lara, H. H.; Mullins, S. M.; Black, D. M.; Ramsower, H. M.; Alvarez, M. M.; Williams, T. L.; Lopez-Lozano, X.; Weissker, H.-C.; García, A. P. et al. Tetrahedral (T) Closed-Shell Cluster of 29 Silver Atoms \& 12 Lipoate Ligands, $\left[\operatorname{Ag}_{29}(\mathrm{R}-\alpha-\mathrm{LA})_{12}\right]^{(3-)}$ : Antibacterial and Antifungal Activity. ACS Applied Nano Materials 2018, 1, 1595-1602.

(7) Negishi, Y.; Nakazaki, T.; Malola, S.; Takano, S.; Niihori, Y.; Kurashige, W.; Yamazoe, S.; Tsukuda, T.; Häkkinen, H. A Critical Size for Emergence of Nonbulk Electronic and Geometric Structures in Dodecanethiolate-Protected Au Clusters. Journal of the American Chemical Society 2015, 137, 1206-1212, PMID: 25549276.

(8) Jin, R.; Pei, Y.; Tsukuda, T. Controlling Nanoparticles with Atomic Precision. Accounts of Chemical Research 2019, 52, 1-1.

(9) Whetten, R. L.; Weissker, H.-C.; Pelayo, J. J.; Mullins, S. M.; López-Lozano, X.; Garzón, I. L. Chiral-Icosahedral (I) Symmetry in Ubiquitous Metallic Cluster Compounds (145A,60X): Structure and Bonding Principles. Accounts of Chemical Research 2019, 52, 34-43.

(10) Yan, N.; Xia, N.; Liao, L.; Zhu, M.; Jin, F.; Jin, R.; Wu, Z. Unraveling the longpursued Au144 structure by x-ray crystallography. Science Advances 2018, 4.

(11) Lei, Z.; Li, J.-J.; Wan, X.-K.; Zhang, W.-H.; Wang, Q.-M. Isolation and Total Structure Determination of an All-Alkynyl-Protected Gold Nanocluster Au144. Angewandte Chemie International Edition 2018, 57, 8639-8643. 
(12) Lopez-Acevedo, O.; Akola, J.; Whetten, R. L.; Grönbeck, H.; Häkkinen, H. Structure and Bonding in the Ubiquitous Icosahedral Metallic Gold Cluster $\mathrm{Au}_{144}(\mathrm{SR})_{60}$. The Journal of Physical Chemistry C 2009, 113, 5035-5038.

(13) Bahena, D.; Bhattarai, N.; Santiago, U.; Tlahuice, A.; Ponce, A.; Bach, S. B. H.; Yoon, B.; Whetten, R. L.; Landman, U.; Jose-Yacaman, M. STEM Electron Diffraction and High-Resolution Images Used in the Determination of the Crystal Structure of the $\mathrm{Au}_{144}(\mathrm{SR})_{60}$ Cluster. The Journal of Physical Chemistry Letters 2013, 4, $975-981$.

(14) Weissker, H.-C.; Escobar, H. B.; Thanthirige, V. D.; Kwak, K.; Lee, D.; Ramakrishna, G.; Whetten, R.; ; López-Lozano, X. Information on quantum states pervades the visible spectrum of the ubiquitous Au144 gold nanocluster. Nature Communications 2014, 5, 3785 .

(15) Weissker, H.-C.; Lopez-Acevedo, O.; Whetten, R. L.; López-Lozano, X. Optical Spectra of the Special Au144 Gold-Cluster Compounds: Sensitivity to Structure and Symmetry. The Journal of Physical Chemistry C 2015, 119, 11250-11259.

(16) Ma, X.; Tang, Z.; Qin, L.; Peng, J.; Li, L.; Chen, S. Unravelling the formation mechanism of alkynyl protected gold clusters: a case study of phenylacetylene stabilized Au144 molecules. Nanoscale 2020, 12, 2980-2986.

(17) Walter, M.; Akola, J.; Lopez-Acevedo, O.; Jadzinsky, P. D.; Calero, G.; Ackerson, C. J.; Whetten, R. L.; Grönbeck, H.; Häkkinen, H. A unified view of ligand-protected gold clusters as superatom complexes. Proceedings of the National Academy of Sciences 2008, 105, 9157-9162.

(18) Malola, S.; Lehtovaara, L.; Enkovaara, J.; Häkkinen, H. Birth of the Localized Surface Plasmon Resonance in Monolayer-Protected Gold Nanoclusters. ACS Nano 2013, \%, 10263-10270.

(19) Barcaro, G.; Sementa, L.; Fortunelli, A.; Stener, M. Comment on " $(\mathrm{Au}-\mathrm{Ag})_{144}(\mathrm{SR})_{60}$ 
alloy nanomolecules" by C. Kumara and A. Dass, Nanoscale, 2011, 3, 3064. Nanoscale 2015, 7, 8166-8167.

(20) Sinha-Roy, R.; López-Lozano, X.; Whetten, R. L.; García-González, P.; Weissker, H.-C. In Search of the Quantum-Electronic Origin of Color Change: Elucidation of the Subtle Effects of Alloying with Copper on $\approx 1.8 \mathrm{~nm}$ Gold Nanoclusters. The Journal of Physical Chemistry C 2017, 121, 5753-5760.

(21) Baseggio, O.; De Vetta, M.; Fronzoni, G.; Toffoli, D.; Stener, M.; Sementa, L.; Fortunelli, A. Time-dependent density-functional study of the photoabsorption spectrum of $\mathrm{Au}_{25}\left(\mathrm{SC}_{2} \mathrm{H}_{4} \mathrm{C}_{6} \mathrm{H}_{5}\right)_{18}$ anion: Validation of the computational protocol. International Journal of Quantum Chemistry 2018, 118, e25769.

(22) Sementa, L.; Barcaro, G.; Dass, A.; Stener, M.; Fortunelli, A. Designing ligandenhanced optical absorption of thiolated gold nanoclusters. Chem. Commun. 2015, $51,7935-7938$.

(23) Rambukwella, M.; Burrage, S.; Neubrander, M.; Baseggio, O.; Aprà, E.; Stener, M.; Fortunelli, A.; Dass, A. $\mathrm{Au}_{38}(\mathrm{SPh})_{24}: \mathrm{Au}_{38}$ Protected with Aromatic Thiolate Ligands. The Journal of Physical Chemistry Letters 2017, 8, 1530-1537.

(24) Theivendran, S.; Chang, L.; Mukherjee, A.; Sementa, L.; Stener, M.; Fortunelli, A.; Dass, A. Principles of Optical Spectroscopy of Aromatic Alloy Nanomolecules: $\mathrm{Au}_{36-x} \mathrm{Ag}_{x}(\mathrm{SPh}-\mathrm{tBu})_{24}$. The Journal of Physical Chemistry $C$ 2018, 122, 45244531.

(25) Sementa, L.; Barcaro, G.; Baseggio, O.; De Vetta, M.; Dass, A.; Aprà, E.; Stener, M.; Fortunelli, A. Ligand-Enhanced Optical Response of Gold Nanomolecules and Its Fragment Projection Analysis: The Case of $\mathrm{Au}_{30}(\mathrm{SR})_{18}$. The Journal of Physical Chemistry C 2017, 121, 10832-10842.

(26) Malola, S.; Kaappa, S.; Häkkinen, H. Role of Nanocrystal Symmetry in the Crossover Region from Molecular to Metallic Gold Nanoparticles. The Journal of Physical Chemistry C 2019, 123, 20655-20663. 
(27) Sinha-Roy, R.; García-González, P.; López Lozano, X.; Whetten, R. L.; Weissker, H.C. Identifying Electronic Modes by Fourier Transform from $\delta$-Kick Time-Evolution TDDFT Calculations. Journal of Chemical Theory and Computation 2018, 14, 6417-6426.

(28) Lopez-Lozano, X.; Barron, H.; Mottet, C.; Weissker, H.-C. Aspect-ratio- and sizedependent emergence of the surface-plasmon resonance in gold nanorods - an ab initio TDDFT study. Phys. Chem. Chem. Phys. 2014, 16, 1820-1823.

(29) Johnson, H. E.; Aikens, C. M. Electronic Structure and TDDFT Optical Absorption Spectra of Silver Nanorods. The Journal of Physical Chemistry A 2009, 113, 44454450.

(30) Sinha-Roy, R.; García-González, P.; Weissker, H.-C.; Rabilloud, F.; FernándezDomínguez, A. I. Classical and ab Initio Plasmonics Meet at Sub-nanometric Noble Metal Rods. ACS Photonics 2017, 4, 1484-1493.

(31) Shen, B.; Tatchen, J.; Sanchez-Garcia, E.; Bettinger, H. F. Evolution of the Optical Gap in the Acene Series: Undecacene. Angewandte Chemie International Edition 2018, 57, 10506-10509. 


\section{Graphical TOC Entry}

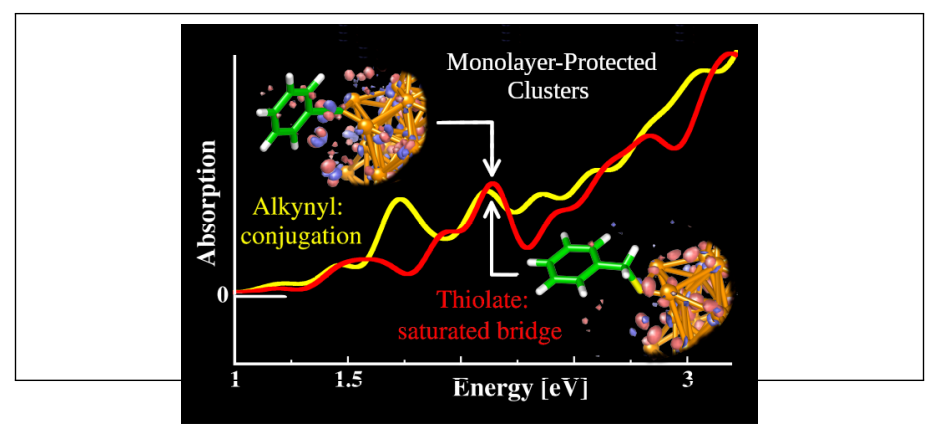

